\title{
La investigación en el aula a través de las redes sociales: co-diseño de un blog como herramienta de innovación y mejora de la docencia.
}

\author{
Llop-Medina, Laura ${ }^{1 a}$; Bueno-Sanchez, Lidia ${ }^{\text {a }}$; Sigalat-Signes, Enric ${ }^{\text {a }}$; Ródenas- \\ Rigla, Francisco ${ }^{a}$. \\ ${ }^{a}$ Universitat de València, Valencia, España. ${ }^{1}$ Autora correspondencia. Correo electrónico: \\ laura.1lop@uv.es
}

\begin{abstract}
Resumen
El nuevo Espacio Europeo de Educación ha supuesto la incorporación de nuevas metodologías y dinámicas digitales en entornos formales como el ámbito universitario, motivando al alumnado a involucrarse en sus procesos de aprendizaje colaborativo dentro del aula. A través de la asignatura Investigación en Sistemas de Bienestar Social del grado de Trabajo Social de la Universitat de València se ha llevado a cabo un proyecto de innovación docente, financiado por dicha universidad, con el objetivo de mejorar la docencia a través del uso de las redes sociales para la construcción e intercambio de conocimiento superior en materia de investigación. Concretamente, se ha co-diseñado junto al alumnado un blog grupal didáctico de acceso libre, "Investiblog", que permite el uso colectivo de un espacio virtual de encuentro, que contribuye a estrechar la brecha digital debido a su bajo coste y sencillez de uso. El proyecto se ha implementado en el curso académico 2019-2020, en tres grupos de tercer curso, con un total de 124 estudiantes, a través de una metodología colaborativa de co-creación de materiales de aprendizaje compartidos. En la fase pre, se administró un cuestionario ad hoc para establecer los conocimientos previos del alumnado sobre el uso de la herramienta blog. Y en la fase post, se les preguntó por la utilidad y el impacto de la herramienta en el proceso de aprendizaje. Los resultados indican que la nueva herramienta impacta positivamente al facilitar compartir contenidos comunes, obtener información de interés y optar a ayudas para iniciarse en la investigación. Respecto al profesorado, Investiblog aumentó su implicación en el desarrollo de nuevas metodologías docentes co-creadas. En conclusión, las herramientas de aprendizaje cocreadas con el alumnado facilitan el aprendizaje colaborativo y estimulan la creatividad, estas herramientas pueden ser muy útiles para la docencia en situaciones como la provocada por la crisis COVID-19.
\end{abstract}


La investigación en el aula a través de las redes sociales: co-diseño de un blog como herramienta de innovación y mejora de la docencia.

Palabras clave: innovación docente, co-diseño, Investiblog, aprendizaje significativo, aprendizaje colaborativo.

\section{Introducción}

En el Espacio Europeo de Educación Superior, dentro del ámbito de la mejora de la calidad de la enseñanza, se propone que el alumnado sea partícipe de su propio aprendizaje de manera activa durante todo el proceso, utilizando para ello modelos de aprendizaje colaborativo como eje central de toda la acción innovadora a través de las TIC.

Los blogs usados con fines educativos o en entornos de aprendizaje son conocidos como Edublogs; aquellos blogs cuyo principal objetivo es apoyar un proceso de enseñanzaaprendizaje en un contexto educativo (Tíscar Lara, 2005). A través del Edublog, se abre un abanico muy amplio de posibilidades, puesto que esta herramienta permite el uso colectivo de un espacio virtual de encuentro, dinamización de grupos, motivación del alumnado, estrechando la brecha digital. Ciertamente, los Edublogs son versátiles, permiten ser usados en un amplio abanico de posibilidades y estrategias de carácter metodológico (Cabero, López y Ballesteros, 2009) como realización y presentación de proyectos grupales, confección de mapas conceptuales, discusiones, conexión con otros sitios webs o el soporte al estudio.

Son muchas las investigaciones en la literatura científica que apuntan que el blog es una herramienta eficaz cuando se hace servir para la mejora de la reflexión (Parkes y Kajder, 2010; Delgado et. al, 2018) y de los procesos de enseñanza-aprendizaje (Deng y Yuen, 2011; 2010; Blanco, 2016; Gámez, Rodríguez y Torres, 2018; Portuguez, 2018), además los estudiantes perciben que logran mayores grados de aprendizaje a través de los blogs (García, Moizer, Wilkins y Haddoud, 2019). Su utilización, se ha acrecentado en el contexto universitario en los últimos años debido al interés que provocan tanto en alumnos como en profesores (Santoveña, 2011; Ifinedo, 2018). Además, entre otras características destacar que propicia la comunicación horizontal, presenta una alta versatilidad, accesibilidad y un bajo o nulo coste -si se trata de un producto libre-.

En este sentido, numerosos trabajos coinciden en que los blogs son herramientas didácticas válidas y convenientes para la adquisición de conocimientos en el contexto universitario señalando el papel activo del alumnado en su proceso de aprendizaje-, que propician el intercambio de ideas, de información y son facilitadores de la comunicación entre los alumnos y el profesor (Top, 2012; García Laborda, 2018, Martín, 2009). El hecho de codiseñar y/o usar un blog no solamente favorece la creatividad, la cuestión es mucho más transcendente, se trata aquí también de facilitar el aprendizaje colaborativo y cooperativo y 
de crear comunidad (Top, 2012). Sin embargo, también hay aspectos negativos que hacen referencia a otras experiencias no tan positivas que tienen que ver básicamente con el abandono (y mortalidad) del blog como herramienta pedagógica (González Sánchez y García Muiña, 2011).

Con todo, parece innegable el potencial que ofrecen los blogs. Se trata de una herramienta facilitadora del transitar de un arquetipo de enseñanza más tradicional al actual entorno europeo de convergencia (Baumgartner, 2004). En esta línea situamos el presente trabajo, se pretende dar respuesta de forma tentativa a una serie de interrogantes: ¿Cómo percibe el alumnado la utilidad del uso de un blog en el contexto académico?, ¿cuál es la opinión de éstos respecto a sus puntos fuertes?, ¿les ha ayudado a desarrollar habilidades y competencias profesionales?, y como cuestión capital a responder: ¿ha resultado útil la utilización de esta herramienta para el aprendizaje y la docencia en situaciones como la provocada por la crisis COVID-19?

En este trabajo se presenta una experiencia de innovación docente interdisciplinar, cuyo objetivo es el de mejorar la docencia a través del uso de las redes sociales para la construcción e intercambio de conocimiento superior en materia de investigación.

\section{Metodología}

El proyecto implementó una metodología colaborativa de co-creación de materiales de aprendizaje compartidos para diseñar un Edublog. Concretamente, se ha co-diseñado junto al alumnado un blog grupal didáctico de acceso libre, "Investiblog", que permite el uso colectivo de un espacio virtual de encuentro alojado en el servidor web de la Universitat de València.

Los contenidos incorporados en "Investiblog" durante el cuatrimestre incluían normas básicas de convivencia, elementos fundamentales para el estudio (mapas conceptuales), batería de preguntas tipo test para la preparación del examen, posters científicos elaborados por el alumnado, gestores bibliográficos y gestores de proyectos de investigación.

\subsection{Muestra}

La muestra del estudio estaba integrada por el total de estudiantes matriculados en el curso académico 2019-2020, en los grupos A, B y G de la asignatura "Investigación en Sistemas de Bienestar Social (código 33502)" del grado en Trabajo Social, que se ha impartido ha impartido bajo una modalidad presencial y online (desde marzo de 2020). En estos grupos había matriculados un total de 124 estudiantes (G34, B45, A45). La participación en la 
La investigación en el aula a través de las redes sociales: co-diseño de un blog como herramienta de innovación y mejora de la docencia.

creación del blog y la respuesta a los cuestionarios de evaluación fue voluntaria, informando al alumnado de que dicha participación no tendría ninguna incidencia en sus calificaciones. En la fase pre el índice de respuesta sobre la muestra fue 0.77 , mientras que en el post fue de 0.62 .

Tabla 2.1. Distribución de la muestra

\begin{tabular}{|c|c|c|c|}
\hline & & Pre (n 95) & Post (n 77) \\
\hline \multirow{3}{*}{ Mujeres } & Frecuencia (\%) & $79(83,2)$ & $64(83,1)$ \\
\hline & Edad Media (DS) & $23(\mathrm{SD}+-7)$ & $22(\mathrm{SD}+-3)$ \\
\hline & Max-Min & $56-20$ & $36-20$ \\
\hline \multirow{3}{*}{ Hombres } & Frecuencia (\%) & $16(16,8)$ & $13(16,9)$ \\
\hline & Edad Media & $24(\mathrm{SD}+-6)$ & $25(\mathrm{SD}+-5)$ \\
\hline & Max-Min & $40-20$ & $38-20$ \\
\hline \multirow{3}{*}{ TOTAL } & Frecuencia (\%) & $95(100)$ & $77(100)$ \\
\hline & \multirow{2}{*}{ Edad Media } & $23(\mathrm{SD}+-7)$ & $23(\mathrm{SD}+-3)$ \\
\hline & & $56-20$ & $38-20$ \\
\hline
\end{tabular}

Fuente: elaboración propia

\subsection{Instrumentos utilizados}

En la fase pre y post se administró un cuestionario ad hoc, utilizando google forms. Ambos instrumentos fueron diseñados por el equipo de investigación.

El instrumento pre, centrado en los conocimientos previos del alumnado sobre el uso de la herramienta blog, contenía 10 ítems, de ellos 7 eran preguntas dicotómicas (sí/no), 2 preguntas con varias opciones excluyentes y una pregunta abierta. El instrumento post, que incidía en la utilidad y el impacto de la herramienta en el proceso de aprendizaje, estaba integrado por 18 ítems, de ellos 5 eran preguntas con varias opciones excluyentes, 9 preguntas incluían una escala Likert con cinco niveles, 2 preguntas dicotómicas (sí/no) y 2 preguntas abiertas. El primer instrumento se pasó al inicio del cuatrimestre, entre el 29 de enero y el 7 de febrero de 2020; y el segundo, se pasó a todos los grupos tras realizar el examen de la asignatura el 5 de junio de 2020.

\subsection{Análisis realizados}

Se han elaborado tablas de frecuencias con los principales datos, aplicando Chi Cuadrado para establecer la independencia entre variables categóricas. Se ha utilizado la aplicación SPSS V26.0.0. 


\section{Resultados}

\subsection{Análisis de cuestionarios}

En el pre, el 69,5\% alumnado (66) manifestó que conocía el funcionamiento de un blog y el $54,3 \%$ (51) indicó que lo habían utilizado en alguna asignatura a lo lardo de su vida académica, de ellos/as el 64,7\% (33) lo utilizó en la enseñanza universitaria. Antes de comenzar a utilizar el blog en la asignatura, un 44,2\% (42) creía que resultaría útil y 53,7\% (51) respondió que no estaba seguro de su utilidad. No existe una relación significativa entre haber utilizado un blog en su vida académica y el nivel de utilidad de esta herramienta $\left(\mathrm{X}^{2}=3.202 ; \mathrm{p}=0.202\right)$.

En el post, el 78,7\% del alumnado (59) señala que utiliza más de $3 \mathrm{~h}$ al día internet y un $53,4 \%$ (39) señala que ese uso está dedicado al estudio. El uso del blog como herramienta de trabajo en el aula tiene un impacto positivo en la percepción del alumnado, ya que consideran la herramienta como un elemento facilitador de información y estudio compartido. Obteniendo mayor relevancia en el periodo de confinamiento por estado de alarma por COVID-19. Concretamente, el 88,3\% de los participantes calificó como muy útil las herramientas tipo blog durante periodos largos de confinamiento, especialmente para el estudio individual y el seguimiento de la asignatura (Tabla 3.1). El alumnado destaca la utilidad del Blog para la preparación del examen $(48,7 \%)$ y a la hora de disponer de recursos que muestren los contenidos de la asignatura de manera más atractiva $(22,4 \%)$. El co-diseño del blog junto al profesorado motivó la participación continua del alumnado y así lo percibieron el 50,7\% de las personas encuestadas. Con todo, el 72,8\% recomendaría el uso del blog en la asignatura en futuros cursos académicos (Tabla 3.2).

Tabla 3.1. Post - Utilidad de Investiblog en confinamiento (frecuencias y porcentajes)

\begin{tabular}{|c|c|c|c|c|c|c|}
\hline $\begin{array}{l}\text { Durante el confinamiento } \\
\text { por el COVID-19 el blog me } \\
\text { ha facilitado }\end{array}$ & $\begin{array}{l}\text { Totalmente } \\
\text { en } \\
\text { desacuerdo }\end{array}$ & $\begin{array}{c}\text { En } \\
\text { desacuerdo }\end{array}$ & Neutral & De acuerdo & $\begin{array}{l}\text { Totalmente } \\
\text { de acuerdo }\end{array}$ & Total \\
\hline Estudio de la asignatura & $\begin{array}{r}6 \\
(7,8 \%)\end{array}$ & $\begin{array}{r}6 \\
(7,8 \%)\end{array}$ & $\begin{array}{r}14 \\
(18,2 \%)\end{array}$ & $\begin{array}{r}26 \\
(33,8 \%)\end{array}$ & $\begin{array}{r}25 \\
(32,5 \%)\end{array}$ & $\begin{array}{r}77 \\
(100,0 \%)\end{array}$ \\
\hline Seguimiento de la asignatura & $\begin{array}{r}7 \\
(9,1 \%)\end{array}$ & $\begin{array}{r}6 \\
(7,8 \%)\end{array}$ & $\begin{array}{r}16 \\
(20,8 \%)\end{array}$ & $\begin{array}{r}25 \\
(32,5 \%)\end{array}$ & $\begin{array}{r}23 \\
(29,9 \%)\end{array}$ & $\begin{array}{r}77 \\
(100,0 \%)\end{array}$ \\
\hline $\begin{array}{l}\text { Comunicación con } \\
\text { compañeras y profesorado. }\end{array}$ & $\begin{array}{r}10 \\
(13,0 \%)\end{array}$ & $\begin{array}{r}12 \\
(15,6 \%)\end{array}$ & $\begin{array}{r}15 \\
(19,5 \%)\end{array}$ & $\begin{array}{r}24 \\
(31,2 \%)\end{array}$ & $\begin{array}{r}16 \\
(20,8 \%)\end{array}$ & $\begin{array}{r}77 \\
(100,0 \%)\end{array}$ \\
\hline
\end{tabular}

Fuente: elaboración propia 
La investigación en el aula a través de las redes sociales: co-diseño de un blog como herramienta de innovación y mejora de la docencia.

Tabla 3.2 Post - Utilidad de Investiblog en los procesos de formación (frecuencias y porcentajes)

\begin{tabular}{|c|c|c|c|c|c|c|}
\hline & $\begin{array}{l}\text { Totalmente } \\
\text { en } \\
\text { desacuerdo }\end{array}$ & $\begin{array}{c}\text { En } \\
\text { desacuerdo }\end{array}$ & Neutral & De acuerdo & $\begin{array}{c}\text { Totalmente de } \\
\text { acuerdo }\end{array}$ & Total \\
\hline $\begin{array}{l}\text { Co-diseñar el } \\
\text { blog me ha } \\
\text { motivado a ser } \\
\text { más participativa }\end{array}$ & $\begin{array}{r}2 \\
(2,6 \%)\end{array}$ & $\begin{array}{r}7 \\
(9,1 \%)\end{array}$ & $\begin{array}{r}29 \\
(37,7 \%)\end{array}$ & $\begin{array}{r}21 \\
(27,3 \%)\end{array}$ & $\begin{array}{r}18 \\
(23,4 \%)\end{array}$ & $\begin{array}{r}77 \\
(100,0 \%)\end{array}$ \\
\hline $\begin{array}{l}\text { Complementa los } \\
\text { contenidos del } \\
\text { aula virtual }\end{array}$ & $\begin{array}{r}3 \\
(3,9 \%)\end{array}$ & $\begin{array}{r}6 \\
(7,8 \%)\end{array}$ & $\begin{array}{r}19 \\
(24,7 \%)\end{array}$ & $\begin{array}{r}29 \\
(37,7 \%)\end{array}$ & $\begin{array}{r}20 \\
(26,0 \%)\end{array}$ & $\begin{array}{r}77 \\
(100,0 \%)\end{array}$ \\
\hline $\begin{array}{l}\text { Recomendaría el } \\
\text { uso del blog para } \\
\text { futuros cursos } \\
\text { académicos }\end{array}$ & $\begin{array}{r}5 \\
(6,5 \%)\end{array}$ & $\begin{array}{r}5 \\
(6,5 \%)\end{array}$ & $\begin{array}{r}11 \\
(14,3 \%)\end{array}$ & $\begin{array}{r}21 \\
(27,3 \%)\end{array}$ & $\begin{array}{r}35 \\
(45,5 \%)\end{array}$ & $\begin{array}{r}77 \\
(100,0 \%)\end{array}$ \\
\hline $\begin{array}{l}\text { Me gustaría } \\
\text { aprender más } \\
\text { sobre el uso del } \\
\text { blog académico }\end{array}$ & $\begin{array}{r}4 \\
(5,2 \%)\end{array}$ & $\begin{array}{r}2 \\
(2,6 \%)\end{array}$ & $\begin{array}{r}21 \\
(27,3 \%)\end{array}$ & $\begin{array}{r}24 \\
(31,2 \%)\end{array}$ & $\begin{array}{r}26 \\
(33,8 \%)\end{array}$ & $\begin{array}{r}77 \\
(100,0 \%)\end{array}$ \\
\hline $\begin{array}{l}\text { Los comentarios } \\
\text { y entradas me } \\
\text { han sido útiles }\end{array}$ & $\begin{array}{r}4 \\
(5,2 \%)\end{array}$ & $\begin{array}{r}14 \\
(18,2 \%)\end{array}$ & $\begin{array}{r}20 \\
(26,0 \%)\end{array}$ & $\begin{array}{r}17 \\
(22,1 \%)\end{array}$ & $\begin{array}{r}22 \\
(28,6 \%)\end{array}$ & $\begin{array}{r}77 \\
(100,0 \%)\end{array}$ \\
\hline
\end{tabular}

Fuente: elaboración propia

Finalmente, se recogieron algunas sugerencias de mejora para futuras versiones de Investiblog. Entre ellas, se destacan las siguientes:

- Recoger experiencias en investigación o relatos en primera persona que sirvan como recomendaciones al alumnado.

- Añadir mayor contenido audiovisual, documentales, salas de debate, noticias interesantes.

- Facilitar la navegación y el uso del contenido.

- Resaltar la importancia al uso del blog durante el transcurso de la asignatura.

- Realizar versiones en las lenguas cooficiales (valenciano)

\section{Conclusiones}

La implementación de Investiblog propició el estudio, el seguimiento y la comprensión de los contenidos de la asignatura, sirviéndoles de ayuda en el proceso de enseñanzaaprendizaje. La valoración de la experiencia de innovación docente ha resultado positiva. En este sentido, 7 de cada 10 alumnos recomendaría el uso de blogs como el diseñado en este proyecto. La participación del alumnado en la toma de decisiones desde el inicio del diseño, así como la participación e implicación dinámica del conjunto del profesorado, estableció un ambiente académico y a la vez distendido, que fomentó la creatividad y 
participación de todas las personas implicadas. De esta manera, se generaron dinámicas de participación continuas y espontáneas, tal y como apuntaban autores como Top, E., (2012) y García, J (2018), que mejoraron el contenido de los debates, las entradas y sobretodo, mejoró la transición de las clases presenciales a la docencia online durante la crisis por la pandemia COVID-19. No obstante, como señalan Verdín, Abundis \& Gónzalez (2020) es importante analizar cómo los docentes incorporan la innovación docente en su práctica profesional dentro y fuera del aula, como sujetos posibilitadores del cambio.

A través de los instrumentos diseñados para recoger la experiencia del alumnado se reunió información relevante sobre las necesidades que presenta actualmente la generación de personas nativas digitales, dotando al profesorado de una información crucial para el desarrollo y adecuación de su quehacer docente en las nuevas sociedades digitales. En fases posteriores se plantea el uso de las tecnologías colaborativas en el diseño de evaluaciones atractivas, en la línea de lo planteado por Bryan \& Clegg (2019), y la aplicación del método de juego de roles a través del uso de la tecnología audiovisual para promover la mejora de las variables de actitud y habilidades prácticas del alumnado, tal y como han mostrado autores como Moreno et al. (2020).

\section{Agradecimientos}

Este proyecto ha sido financiado por el Servei de Formació Permanent i Innovació Educativa de la Universitat de Valencia, dentro del "Programa de Innovación Docente, 2019” (ref. UV-SFPIE_PID19-1096293).

\section{Referencias}

Baumgartner, P. (2004). The Zen Art of Teaching. Communication and Interactions in Eeducation. In the International Workshop ICL2004, Austria.

Blanco García, A. I. (2016). El uso de blogs en la innovación docente: un nuevo paradigma de enseñanza-aprendizaje. Actualidad Jurídica Iberoamericana, 4(2): 27 - 44.

Bryan, C., \& Clegg, K. (Eds.). (2019). Innovative assessment in higher education: A handbook for academic practitioners. Routledge.

Cabero, J., López, E., \& Ballesteros, C. (2009). Experiencias universitarias innovadoras con blogs para la mejora de la praxis educativa en el contexto europeo. RUSC. Revista De Universidad Y Sociedad Del Conocimiento, vol.6, n.2. http://www.raco.cat/index.php/RUSC/article/view/140242/191428

Delgado, V., Hortigüela, D., Ausín, V., \& Abella, V. (2018). El Blog como instrumento de mejora para la autorregulación del aprendizaje del estudiante universitario. Estudios pedagógicos (Valdivia), 44(2), 171-184. 
La investigación en el aula a través de las redes sociales: co-diseño de un blog como herramienta de innovación y mejora de la docencia.

Deng, L., \& Yuen, A. H. (2011). Towards a framework for educational affordances of blogs. Computers \& education, 56(2), 441-451.

Gámez, F. I. L., Rodríguez, M. R., \& Torres, L. E. S. (2018). Uso y aplicación de las TIC en el proceso de enseñanza-aprendizaje. Revista Científica de FAREM-Estelí, (25), 16-30.

Garcia, E., Moizer, J., Wilkins, S., \& Haddoud, M. Y. (2019). Student learning in higher education through blogging in the classroom. Computers \& Education, 136, 61-74.

García Laborda, J. (2018, September). Razones para el uso de blogs por maestros en formación. In IN-RED 2018. IV Congreso Nacional de Innovación Educativa y Docencia en Red (pp. 16-22). Editorial Universitat Politècnica de València.

González Sánchez, R., \& García Muiña, F.E. (2011). Recursos eficaces para el aprendizaje en entornos virtuales en el Espacio Europeo de Educación Superior: análisis de los edublogs. ESE. Estudios sobre educación, vol.20, 161-180.

Ifinedo, P. (2018). Determinants of students' continuance intention to use blogs to learn: An empirical investigation. Behaviour \& Information Technology, 37(4), 381-392.

Martín, O. (2009). Educar en comunidad: promesas y realidades de la Web 2.0 para la innovación pedagógica. En Carneiro, R.; Toscano, J.C y Díaz, T. (coords.): Los desafíos de las TIC para el cambio educativo. Madrid: colección Metas Educativas 2021, OEI- Fundación Santillana.

Moreno-Guerrero, A. J., Rodríguez-Jiménez, C., Gómez-García, G., \& Ramos Navas-Parejo, M. (2020). Educational Innovation in Higher Education: Use of Role Playing and Educational Video in Future Teachers' Training. Sustainability, 12(6), 2558.

Parkes, K., \& Kajder, S. (2010). Eliciting and assessing reflective practice: A case study in web 2.0 technologies. International Journal of Teaching and Learning in Higher Education, vol.22, n.2, 218-228

Portuguez Castro, M. (2018). Uso de blogs educativos para lograr aprendizajes significativos: Una propuesta para la educación a distancia en Costa Rica.

Santoveña, S. (2011). Incidencia de los nuevos alfabetismos en la mejora de la calidad de la enseñanza: El caso de los blogs. Aula Abierta, vol.39, n.2, 59-68.

Tíscar, Lara. (2005). Weblogs y Educación. Consultado 28 de junio, 2019 desde http://tiscar.com/weblogs-y-educacion.

Top, E. (2012). Blogging as a social medium in undergraduate courses: Sense of community best predictor of perceived learning. The Internet and Higher Education, 15(1), 24-28.

Verdín-Zea, A. A., Abundis-De León, F., \& Gonzalez-Basilio, S. D. J. (2020). Educational innovation and university academics: the challenges of incorporating change into teaching and management practice. Journal of Human Resources, 6(17), 1-9. 